\title{
PAPEL DE LA INMUNOLOGÍA EN LA ENDOCRINOLOGÍA GINECOLÓGICA: REVISIÓN DE LA LITERATURA
}

\author{
The role of immunology in gynaecological \\ end ocrinology: a literature review \\ Ivonne Díaz-Yamal, M.D. *, Carolina Ruiz-Wagner, M.D. ** \\ Recibido: julio 1/09 - Aceptado: octubre 26/09
}

\section{RESUMEN}

Objetivos: revisar la asociación que existe entre los diferentes mecanismos inmunes y los eventos endocrinos necesarios para la regulación de la reproducción humana.

Metodología: el presente trabajo es una revisión sistemática cualitativa, para la cual se consultaron las bases de datos de Ovid y PubMed/Medline, seleccionando artículos publicados entre 1984 y 2009 referentes a estudios en animales y humanos que demostraran la relación existente entre los esteroides y la hormona liberadora de gonadotropinas con el sistema inmune, así como su expresión en algunas enfermedades de tipo autoinmune.

Resultados: muchos estudios experimentales en animales han demostrado una asociación entre el sistema inmune y los diferentes ejes neuroendocrinos que regulan la reproducción humana. Aunque en el hombre no han sido dilucidados los mecanismos definitivos, se ha observado que la Hormona Liberadora de Gonadotropinas (Gonadotropin-Releasing

* Especialista en Ginecología y Obstetricia. Docente de la Unidad de Fertilidad y Endocrinología Reproductiva, Hospital Militar Central, Universidad Militar Nueva Granada. Unidad de Fertilidad por Procreación Medicamente Asistida (PMA), Clínica Marly. Bogotá (Colombia).

** Residente de 3er año de la Universidad Militar Nueva Granada, Bogotá (Colombia). Rotación por Unidad de Fertilidad y Endocrinología Reproductiva, Hospital Militar Central. Bogotá (Colombia). Correo electrónico: ncrwagner@hotmail.com
Hormone [GnRH]) y los esteroides sexuales tienen efectos a nivel de la programación del sistema inmune y su modulación. De la misma forma, se advierte que sistemas inmunes como el de la interleucina-1 juegan un papel importante en la regulación del eje hipotálamo-hipófisis-gónada y de otros ejes involucrados en la reproducción.

Conclusiones: la GnRH y los esteroides son moduladores de la respuesta inmune de manera que pueden estimularla o suprimirla. Los sistemas inmunes como el de interleucinas (IL-1) tienen efectos antigonadotróficos a nivel central, y esteroidogénicos a nivel ovárico, relacionándose así con otros sistemas como el adrenal y el tiroideo con respecto a la ovulación y a la formación del cuerpo lúteo y su regresión; procesos importantes en la reproducción.

Palabras clave: GnRH, estrógenos, andrógenos, citoquinas, interleucinas, quimoquinas, linfocitos, ovulación, prostaglandinas.

\section{SUMMARY}

Objectives: reviewing the association between different immune mechanisms and endocrine events necessary for regulating human reproduction.

Methodology: this was a qualitative systematic review which involved consulting databases like OVID, Medline (Pubmed-MeSH), selecting articles from 1984 to 2009 referring to animal and human 
studies demonstrating the relationship of steroids and the gonadotrophin-releasing hormone (GnRH) with the immune system, as well as their expression in some autoimmune illnesses.

Results: many experimental studies in animals have demonstrated an association among the immune system and different neuroendocrine axes regulating human reproduction. Even though the definitive mechanisms have not yet been elucidated in humans, it has been found that GnRH and sexual steroids have an effect at immune system programming level and on their modulation. Interleukin-1 also plays an important role in regulating the hypothalamushypophysis-gonad axis and other axes involved in the reproduction.

Conclusions: the sexual steroids and GnRH are immune response modulators in such a way that they may stimulate it or have an immunosuppressor role. Immune systems like interleukin (IL-1) have antigonadotropic action at central level and a steroidogenic effect at ovarian level; they are related to the adrenal axis and thyroid activity, ovulation, luteous body formation and regression, all being important processes in reproduction.

Key words: GnRH, oestrogen, androgen, cytokine, interleukin, chemokine, lymphocyte, ovulation, prostaglandin.

\section{INTRODUCCIÓN}

La interacción entre los sistemas endocrino, nervioso e inmune ha sido ampliamente estudiada en trabajos experimentales principalmente en animales. ${ }^{1}$ Por el contrario, en el hombre, los mecanismos definitivos no han sido dilucidados pero se cree que existe una influencia del eje hipotálamo-hipófisisgónada (Hormona Liberadora de Gonadotropinas y esteroides sexuales) en la regulación del sistema inmune y una respuesta género-específica, considerando que enfermedades como el Lupus Eritematoso Sistémico tiene una mayor incidencia en la población femenina. ${ }^{1}$

Así pues, el sistema inmune tiene un importante papel en la regulación de los diferentes ejes (hipotálamo-hipófisis-gónada, hipotálamo-hipófisisadrenal, hipotálamo-hipófisis-tiroides), siendo el sistema de interleucinas, principalmente IL-1, el mejor estudiado con funciones de feedback negativo a diferentes niveles y localmente en el ovario, lo cual evidencia cómo la reproducción humana está ligada con la respuesta inmunitaria. ${ }^{2,3}$

Por lo tanto, el objetivo del presente documento es revisar la asociación existente entre los diferentes mecanismos inmunes con los eventos endocrinos necesarios para la regulación de la reproducción humana. Lo anterior, a partir de la evidencia que actualmente existe acerca de la acción de los mecanismos endocrinos en el sistema inmune, el efecto de las citoquinas en el sistema endocrino, las interacciones entre los sistemas inmune, endocrino y nervioso; y por último, la asociación entre la respuesta inmune con la falla ovárica y la endometriosis.

\section{METODOLOGÍA}

\section{Estrategia de búsqueda}

Se seleccionaron las siguientes palabras clave: "GnRH”, "estrógenos”, “andrógenos”, “citoquinas”, "interleucinas", "quimoquinas", "linfocitos”, "ovulación”, "prostaglandinas”. Acto seguido, se realizó una búsqueda electrónica en las bases de datos de Ovid y PubMed/ Medline, seleccionando artículos publicados entre 1984 y 2009 para identificar la literatura relevante, así como una búsqueda de la literatura en algunos textos de endocrinología ginecológica.

\section{Criterios de selección}

Se incluyeron aquellos artículos referentes a estudios en animales y humanos que demostraran la relación existente entre los esteroides y la hormona liberadora de gonadotropinas con el sistema inmune, así como su expresión en algunas enfermedades de tipo autoinmune.

\section{RESULTADOS}

En total, se obtuvieron 53 artículos con los criterios de selección previamente descritos. A continuación se presenta la evidencia encontrada. 


\section{GnRH, esteroides sexuales y sistema inmune}

Se han reportado sitios de unión específicos para GnRH en cultivos de linfocitos de porcinos ${ }^{2}$ y en timo ${ }^{3}$ y bazo de ratas. ${ }^{4}$ La GnRH tímica idéntica a la hipotalámica en ratas recién nacidas fue reportada por primera vez en 1992, ${ }^{5}$ y la expresión del ARN mensajero (mRNA, por sus siglas en inglés) de GnRH fue demostrada en células inmunes de porcinos (timo, bazo, linfocitos periféricos) en 1997. ${ }^{6}$ En contraste, en humanos la presencia de GnRH inmunorreactiva y bioactiva en células $T$ periféricas $(\mathrm{CD} 4, \mathrm{CD} 8)^{7}$ y linfocitos $\mathrm{B}$ periféricos regula la proliferación de células inmunes sugiriendo una función autocrina. ${ }^{8}$ Asimismo, ésta se ha involucrado en la maduración del timo ${ }^{9}$ y en la programación prenatal y postnatal de células inmunes animales. ${ }^{10} \mathrm{La}$ timopoyesis es esencial para el establecimiento de células $T$ en la vida temprana y adulta, siendo más importante en condiciones de deficiencia inmune tales como infección por VIH, trasplante de médula ósea y períodos de leucopenia luego de altas dosis de quimioterapia cuando la reconstitución inmune es requerida. ${ }^{11}$

En resumen, los estudios en humanos y roedores demuestran que la GnRH:

1. Estimula los receptores de IL-2 para la proliferación y/o activación de células $\mathrm{T}$ y $\mathrm{B}$.

2. Aumenta la producción de interferón gama por las células periféricas mononucleares. ${ }^{12}$

3. Juega un papel relevante en la programación prenatal y postnatal de células inmunes.

En cuanto a los esteroides sexuales, se ha encontrado que éstos influyen en el desarrollo de la inmunidad celular en tejidos linfoides primarios (médula ósea y timo); y adicionalmente, tienen efectos inmunomoduladores en células T y B periféricas. En humanos, se han encontrado receptores de andrógenos (ARs, por sus siglas en inglés) y de estrógenos (ER, por sus siglas en inglés) en órganos linfoides primarios; $;^{13,14}$ y a nivel periférico, sólo receptores de estrógenos. ${ }^{15,16}$
Además de lo anterior, los andrógenos ejercen efectos considerables en el tamaño y la composición del timo, disminuyendo la proliferación y aumentando la apoptosis celular. ${ }^{17}$ En el caso de los receptores de estrógeno ER-beta, estos son responsables de:

1. La formación de células B en la medula ósea. ${ }^{18}$

2. Estimular las células CD4+ y CD8+.

3. Activar una vía extratímica de autoreactivación de la diferenciación de células T en el hígado. ${ }^{19}$

4. A nivel periférico en las células B, aumentar el número de anticuerpos y los niveles de autoanticuerpos circulantes sin aumentar el número de células. ${ }^{20}$

En general, los andrógenos inhiben la respuesta inmune mientras los estrógenos estimulan la inmunidad celular y humoral.

Esta podría ser la razón por la cual los desórdenes autoinmunes son más comunes en las mujeres, de ahí que los esteroides sexuales cumplan un papel importante en la respuesta dimórfica sexual del sistema inmune. Ahora bien, la relación entre los esteroides sexuales y la autoinmunidad ha sido estudiada en modelos de ratones con lupus eritematoso sistémico, diabetes insulino-dependientes y tiroiditis, ${ }^{21}$ encontrando que el estradiol incrementa la producción de autoanticuerpos (IgG anticardiolipina y anti DNA) y acelera la progresión de la respuesta inmune humoral autoinmune activando la vía de la TH2, mientras que los andrógenos tienen un efecto protector. $^{21}$

De otra parte, aún no es claro si la GnRH tiene efectos directos en las células inmunes por estímulo local de su receptor o si actúa indirectamente con la activación del eje hipotálamo-hipófisis-gónada con aumento de los esteroides sexuales; sin embargo, se considera que los estrógenos ejercen un efecto estimulante en las células B y un aumento en niveles de IgG en presencia de GnRH pero no en su ausencia. La testosterona, por su parte, ejerce un efecto supresor en células B en presencia de GnRH pero no en su ausencia, mientras que ambos tipos de esteroides ejercen efectos divergentes en células $\mathrm{T}$ independientemente de la presencia o ausencia de GnRH. ${ }^{22}$ Tabla 1. 


\section{Tabla 1. Efecto de la GnRH y los esteroides sexuales en la respuesta inmune.}

\begin{tabular}{|l|l|l|l|}
\hline \multicolumn{1}{|c|}{ ACCIÓN } & \multicolumn{1}{|c|}{ GnRH } & \multicolumn{1}{c|}{ ESTRÓGENOS } & \multicolumn{1}{c|}{ ANDRÓGENOS } \\
\hline Expresión de receptores. & $\begin{array}{l}\text { En órganos linfoides } \\
\text { primarios y células } \\
\text { periféricas. }\end{array}$ & $\begin{array}{l}\text { En órganos linfoides } \\
\text { primarios y células } \\
\text { periféricas. }\end{array}$ & $\begin{array}{l}\text { En células periféricas } \\
\text { maduras. }\end{array}$ \\
\hline $\begin{array}{l}\text { Efecto en órganos linfoides } \\
\text { primarios. }\end{array}$ & $\begin{array}{l}\text { Involucrado en el desarrollo } \\
\text { y la programación del } \\
\text { sistema inmune. }\end{array}$ & $\begin{array}{l}\text { Regula la formación de } \\
\text { células B en la médula ósea. }\end{array}$ & $\begin{array}{l}\text { Atrofia timo. } \\
\text { Apoptosis timocitos y } \\
\text { precursores de células B } \\
\text { en la médula ósea. }\end{array}$ \\
\hline $\begin{array}{l}\text { Efecto en células inmunes } \\
\text { periféricas }\end{array}$ & $\begin{array}{l}\text { Aumenta los receptores IL- } \\
\text { 2, FNT y células T Helper. }\end{array}$ & $\begin{array}{l}\text { Helper. } \\
\text { Induce la respuesta } \\
\text { humoral por células B. }\end{array}$ & $\begin{array}{l}\text { Estimula las células T } \\
\text { supresoras. Disminuye el } \\
\text { número de células B. }\end{array}$ \\
\hline Impacto en autoinmunidad. & $\begin{array}{l}\text { Exacerba las enfermedades } \\
\text { autoinmunes. }\end{array}$ & $\begin{array}{l}\text { Exacerba las enfermedades } \\
\text { autoinmunes. }\end{array}$ & $\begin{array}{l}\text { Supresor de las enfermeda- } \\
\text { des autoinmunes. }\end{array}$ \\
\hline
\end{tabular}

\section{Citoquinas y sistema endocrino}

Las citoquinas cumplen funciones autocrinas y paracrinas regulando la función inmune, metabólica y endocrina. Lo anterior ocurre principalmente al interactuar con los ejes hipotálamo-hipófisis-gónada, hipotálamohipófisis-adrenal, hipotálamo-hipófisis-tiroides a nivel local en el ovario y el endometrio como mediadores en los diferentes procesos de la reproducción humana. ${ }^{23,24}$ Actualmente, el sistema de la interleucina 1 (IL-1) es el que mejor caracterizado está y su relación con la función reproductiva ha sido descrita claramente. ${ }^{25}$

\section{Efecto a nivel del eje hipotálamo- hipófisis-gónada}

Existen receptores para (IL-1) en las membranas plasmáticas en células $\mathrm{T}$, neuronas hipotalámicas y células B. ${ }^{26-28}$ Varios autores han estudiado los efectos de esta citoquina en el eje hipotálamohipófisis-gónada en modelos animales de ratas in vivo e in vitro, ${ }^{29}$ hallando que la administración intracerebroventricular de IL-1:

1. Inhibe la liberación de la hormona luteinizante (LH, por sus siglas en inglés) con una especificidad suficiente para excluir un efecto en la secreción de la hormona folículo-estimulante (FSH, por sus siglas en inglés). ${ }^{30}$
2. Se asocia con un incremento en los niveles de prolactina y la hormona de crecimiento, ${ }^{31}$ lo cual evidencia el efecto antigonadotrófico de esta interleucina. ${ }^{32,33}$

3. A nivel gonadal existen receptores los cuales permiten una función esteroidogénica bajo condiciones experimentales ${ }^{34}$ pero en humanos aún es controversial ya que los monocitos en sangre periférica y los macrófagos peritoneales son capaces de estimular la producción de progesterona por las células de la granulosa luteinizada. ${ }^{35}$

4. Modula la secreción de gonadotropinas y esteroides gonadales y viceversa.

Se han descrito varias explicaciones acerca de la regulación hormonal del sistema inmune ya que se ha evidenciado que los linfocitos humanos secretan (LH), hormona folículo-estimulante (FSH) o fracción beta-específica de la gonadotrofina coriónica humana (BHCG, por sus siglas en inglés) inmunorreactiva y bioactiva, ${ }^{36}$ las gonadotropinas regulan el mRNA de IL-1 expresados en células hematopoyéticas, la preovulación dependiente de gonadotropinas induce la trascripción de IL-1 en células teca intersticiales en murinos y humanos ${ }^{37}$ y la producción monocítica humana de IL-1 beta es regulada por esteroides gonadales. ${ }^{38}$ Figura 1. 
Figura 1. Efecto de la IL-1 en el eje hipotálamo-hipófisis-gónada.

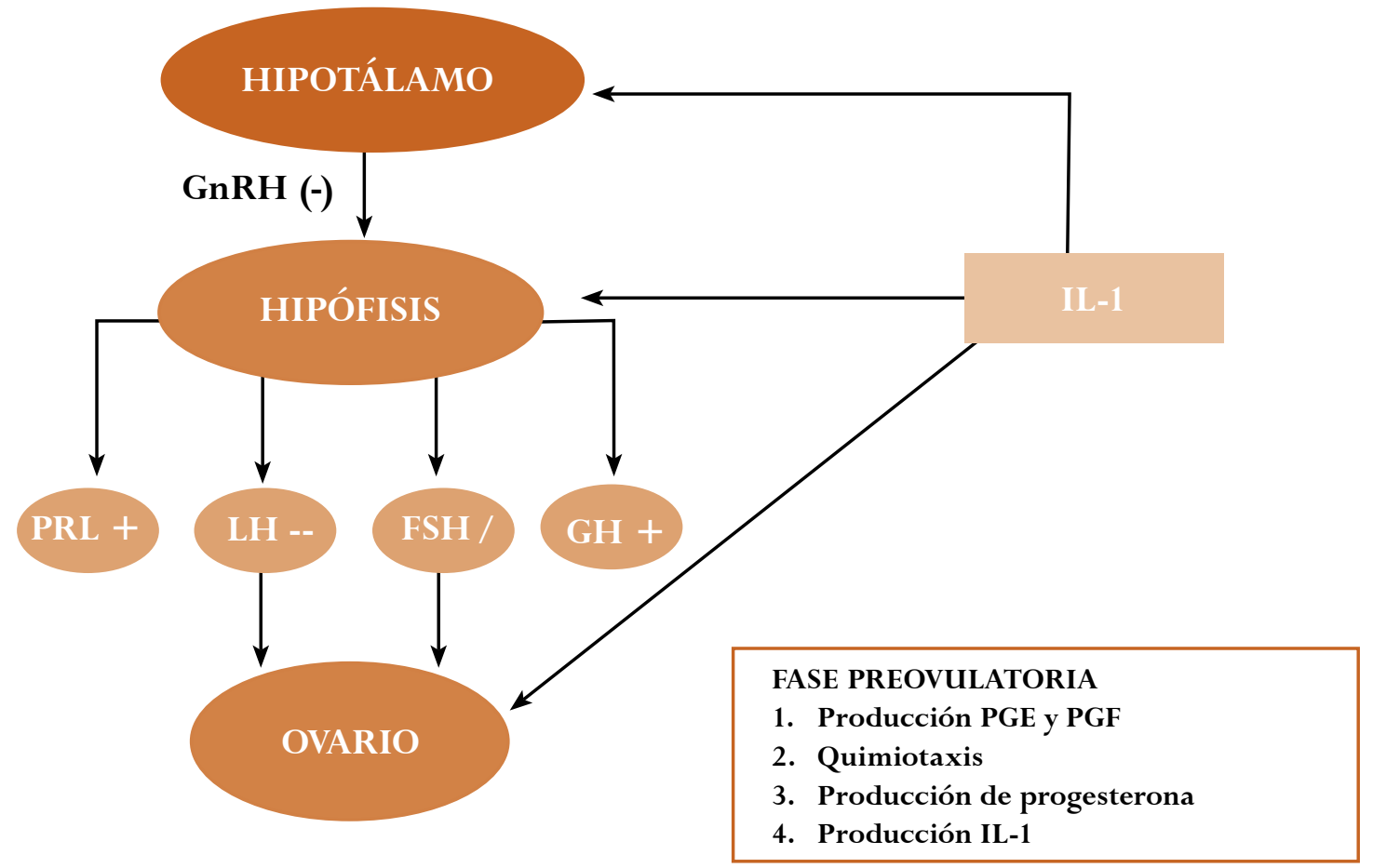

\section{Efecto a nivel del eje hipotálamo- hipófisis-suprarrenal}

Se sabe que la hiperfunción e hipofunción de la glándula suprarrenal resulta en anovulación crónica y amenorrea. El sistema IL-1 activa el eje hipotálamo-hipófisis-adrenal a diferentes niveles. ${ }^{39}$ Asimismo, la IL-1 produce un aumento en la hormona liberadora de corticotropina ( $\mathrm{CRH}$, por sus siglas en inglés) a nivel hipotalámico con la posterior liberación de la hormona adrenocorticotropa (ACTH, por sus siglas en inglés) y de glucocorticoides, ${ }^{40} \mathrm{y}$ en la corteza adrenal, estimula la producción de glucocorticoides. De forma inversa, los glucocorticoides inhiben la excesiva activación del eje y modulan muchas actividades inmunes incluyendo la secreción de IL-1. ${ }^{41}$

\section{Efecto a Nivel del Eje Hipotálamo- Hipófisis-Tiroides}

Las enfermedades tiroideas comunes en mujeres interfieren con la función reproductiva tanto en falla reproductiva como en anovulación o pérdida del embarazo.
Estudios in vitro muestran que la IL-1 inhibe el eje hipotálamo-hipófisis-tiroides al suprimir la secreción de la hormona estimulante de la tiroides (TSH, por sus siglas en inglés), ${ }^{42}$ la biosíntesis y la liberación de hormonas tiroideas y el crecimiento de las células tiroideas, especialmente en altas dosis. ${ }^{43}$ Figura 2.

\section{Efecto a nivel ovárico}

En cuanto al ovario, se sabe que la IL-1 estimula la producción de prostaglandinas (principalmente la $\mathrm{E}$ y F) necesarias en el proceso de ovulación, la atresia y la función del cuerpo lúteo durante las fases preovulatoria y lútea. ${ }^{44}$ Subtipos de células inmunes son reclutadas por quimoquinas foliculares permitiendo la liberación de enzimas proteolíticas, citoquinas, radicales libres y otros mediadores apoptoicos para la ovulación, la neovascularización (luteinización) y la regresión del cuerpo lúteo. El rescate del cuerpo lúteo se da por la disminución de macrófagos en embarazo temprano, lo cual implica a la progesterona como una inhibidora de la respuesta inmune-celular. ${ }^{45}$ 
Figura 2. Efecto de la IL-1 en el eje hipotálamo-hipófisis-suprarrenal-tiroides.

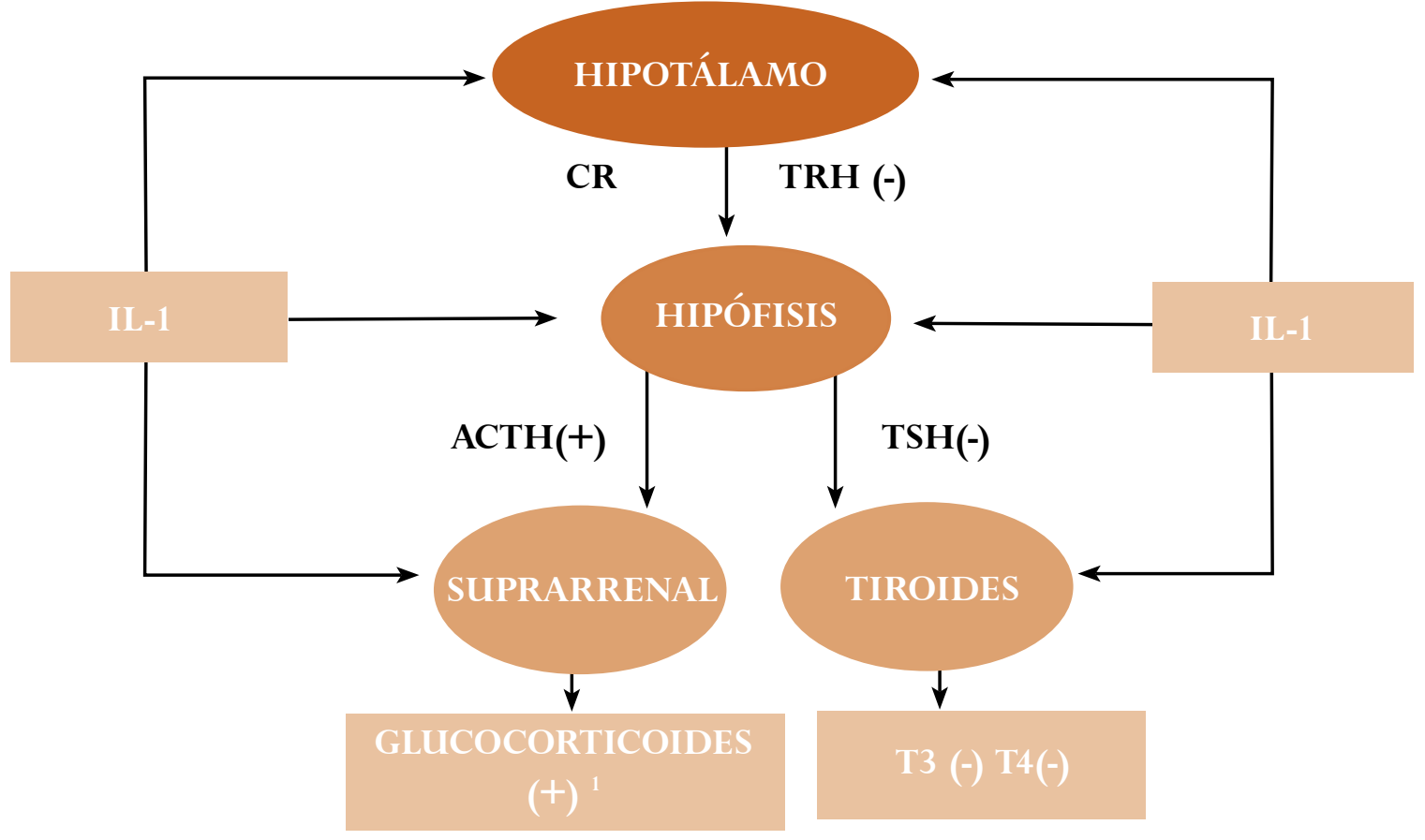

1. Inhiben el eje a nivel hipotalámico, hipofisiario y la producción de IL-1.

\section{Interacción inmune-endocrino- neurológica}

El control de las funciones endocrinas e inmunes van de la mano con la expresión de otros tipos de hormonas metabólicas y factores de crecimiento como la leptina y la grelina, estados de estrés y el sistema nervioso simpático, principalmente. ${ }^{46} \mathrm{La}$ hormona de crecimiento (GH, por sus siglas en inglés) junto con el factor de crecimiento insulinoide tipo I (IGF-1) median la proliferación de células como los condrocitos, fibroblastos, adipositos, mioblastos y linfocitos. ${ }^{47}$ La leptina, en cambio, ejerce un efecto modulador de las respuestas inmunes e inflamatorias al igual que una hormona-citoquina; ${ }^{48}$ y a nivel reproductivo, en niveles bajos, permite el aumento del neuropéptido Y (NPY) en las células hipotalámicas, el cual en presencia de estrógenos puede estimular la liberación de GnRH y gonadotropinas, pero en su ausencia es inhibitorio. ${ }^{49}$ En contraste, la grelina posee efectos inhibitorios ante la respuesta inmune en la expresión de citoquinas proinflamatorias. ${ }^{50}$ En estados de estrés, la activación del eje hipotálamo-hipófisis-adrenal con liberación de glucocorticoides y catecolaminas en el sistema nervioso simpático impactan sobre la función inmune, reduciendo la actividad de las células Natural Killer (NK) la respuesta humoral. ${ }^{51}$ A nivel del eje hipotálamo-hipófisis-gónada hay un efecto inhibitorio por parte de los productos de la proopiomelanocortina (POMC), las endorfinas y los opioides.

\section{Endometriosis y falla ovárica}

Las enfermedades como la endometriosis, caracterizada por la proliferación y funcionamiento de células endometriales ectópicas, se asocia con una alta producción de anticuerpos anormales de tipo IgG e IgA contra el tejido endometrial y ovárico, una disminución en el reconocimiento de antígenos endometriales autólogos, una reducción de 
la citotoxicidad en las células Natural Killer y una resistencia de las células endometriales ectópicas a su destrucción. ${ }^{52}$ En la falla ovárica prematura se encuentran anticuerpos contra células esteroideas, autoanticuerpos contra enzimas como la 17-alfa hidroxilasa, anticuerpos para el receptor de la FSH, y en 50\%, autoanticuerpos para el receptor de LH y contra la zona pelúcida. Además, se han hallado anticuerpos ováricos en 50\%-60\% de mujeres con infertilidad inexplicada y en 60\%-70\% de las pacientes con pobre respuesta a hiperestimulación con gonadotropinas. ${ }^{53}$

\section{CONCLUSIONES}

Los diferentes estudios experimentales muestran como la GnRH y los esteroides sexuales son importantes en el desarrollo, la programación y la modulación del sistema inmune; y juegan un rol en la patogénesis de los desórdenes autoinmunes de tipo género-dependiente. Así pues, la GnRH cumple un papel estimulante en la respuesta inmune, los estrógenos estimulan la respuesta humoral inmune activando desórdenes autoinmunes y los andrógenos tienen una actividad supresora de células T. Claramente, el sistema inmune tiene un rol autocrino, paracrino y endocrino en la regulación de la reproducción humana en eventos como la ovulación, la luteinización y la implantación. Un ejemplo claro de esto es el sistema de citoquinas que modula los diferentes ejes endocrinos a distintos niveles.

\section{REFERENCIAS}

1. Tanriverdi F, Silveira LF, MacColl GS, Bouloux PM. The hypothalamic-pituitary-gonadal axis: immune function and autoimmunity. Endocrinol 2003;176:293-304.

2. Standaert FE, Chew BP, De Avila D, Reeves JJ. Presence of luteinizing hormone-releasing hormone binding sites in cultured porcine lymphocytes. Biol Reprod 1992;46:997-1000.

3. Marchetti B, Guarcello V, Morale MC, Bartoloni G, Farinella Z, Cordaro S, et al. Luteinizing hormonereleasing hormone-binding sites in the rat thymus: characteristics and biological function. Endocrinology 1989;125:1025-36.
4. Batticane N, Morale MC, Gallo F, Farinella Z, Marchetti B. Luteinizing hormone-releasing hormone signaling at the lymphocyte involves stimulation of interleukin-2 receptor expression. Endocrinology 1991;129:277-86.

5. Maier CC, Marchetti B, LeBoeuf RD, Blalock JE. Thymocytes express a mRNA that is identical to hypothalamic luteinizing hormone-releasing hormone mRNA. Cell Mol Neurobiol 1992;12:447-54.

6. Weesner GD, Becker BA, Matteri RL. Expression of luteinizing hormone-releasing hormone and its receptor in porcine immune tissues. Life Sciences 1997;61:1643-9

7. Azad N, La Paglia N, Jurgens KA, Kirsteins L, Emanuele NV, Kelley MR, et al. Immunoactivation enhances the concentration of luteinizing hormonereleasing hormone peptide and its gene expression in human peripheral T-lymphocytes. Endocrinology 1993;133:215-23.

8. Chen HF, Jeung EB, Stephenson M, Leung PC. Human peripheral blood mononuclear cells express gonadotropin-releasing hormone (GnRH), GnRH receptor, and interleukin-2 receptor gamma-chain messenger ribonucleic acids that are regulated by $\mathrm{GnRH}$ in vitro. Clin Endocrinol Metab 1999;84:743-50.

9. Morale MC, Batticane N, Bartoloni G, Guarcello V, Farinella Z, Galasso MG, et al. Blockade of central and peripheral luteinizing hormone-releasing hormone (LHRH) receptors in neonatal rats with a potent LHRH-antagonist inhibits the morphofunctional development of the thymus and maturation of the cell-mediated and humoral immune responses. Endocrinology 1991;128:1073-85.

10. Zakharova LA, Malyukova IV, Adamskaya EI, Kuznetsova TA, Shishkina IV. Luteinizing hormonereleasing hormone in thymus and hypothalamus of rat fetuses: suppressing effect of antagonist and of antibodies on concanavalin A-induced proliferation of thymocytes. Biochemistry (Mosc) 2000;65:1135-9.

11. Flores KG, Li J, Sempowski GD, Haynes BF \& Hale LP. Analysis of the human thymic perivascular space during aging. Clin Invest 1999;104:1031-9.

12. Grasso G, Massai L, De Leo V, Muscettola M. The effect of LHRH and TRH on human interferongamma production in vivo and in vitro. Life Sciences 1998;62:2005-14.

13. Kovacs WJ, Olsen NJ. Androgen receptors in human thymocytes. J Immunol 1987;139:490-3. 
14. Nilsson B, Bergqvist A, Lindblom D, Ljungberg O, Södergård R, von Schoultz B. Characterization and localization of specific oestrogen binding in the human thymus. Gynecol Obstet Invest 1986;21:150-7.

15. Suenaga R, Evans MJ, Mitamura K, Rider V, Abdou NI. Peripheral blood T cells and monocytes and B cell lines derived from patients with lupus express estrogen receptor transcripts similar to those of normal cells. J Rheumat 1998;25:1305-12.

16. Olsen NJ, Kovacs WJ. Effects of androgens on $\mathrm{T}$ and B lymphocyte development. Immunologic Research 2001;23:281-8.

17. Olsen NJ, Olson G, Viselli SM, Gu X, Kovacs WJ. Androgen receptors in thymic epithelium modulate thymus size and thymocyte development. Endocrinology 2001;142:1278-83.

18. Kincade PW, Medina KL, Payne KJ, Rossi MI, Tudor KS, Yamashita Y, et al. Early B-lymphocyte precursors and their regulation by sex steroids. Immunological Reviews 2000;175:128-37.

19. Okuyama R, Abo T, Seki S, Ohteki T, Sugiura K, Kusumi A, et al. Estrogen administration activates extrathymic $\mathrm{T}$ cell differentiation in the liver. J Experiment Med 1992;175:661-9.

20. Verthelyi D, Ansar Ahmed AS. Characterization of estrogen-induced autoantibodies to cardiolipin in nonautoimmune mice. J Autoimmun 1997;10:115-25.

21. Ahmed SA, Verthelyi D. Antibodies to cardiolipin in normal C57BL/6J mice: induction by estrogen but not dihydrotestosterone. J Autoimmun 1993;6:265-79.

22. Jacobson JD, Ansari MD. Immunomodulatory actions of gonadal steroids may be mediated by gonadotropinreleasing hormone. Endocrinology 2004;145:330-6.

23. Simón C, Polan ML. Cytokines and reproduction. West J Med 1994;160:425-9.

24. Arai KI, Lee F, Miyajima A, Miyatake S, Arai N, Yokota T. Cytokines: coordinators of immune and inflammatory responses. Annu Rev Biochem 1990;59:783-836.

25. Dinarello CA. Interleukin-1 and its biologically related cytokines. Adv Immunol 1989;44:153-205.

26. Dower SK, Kronheim SR, Hopp TP, Cantrell M, Deeley M, Gillis S, et al. The cell surface receptors for interleukin-1 alpha and interleukin-1 beta are identical. Nature 1986;324:266-8.

27. Sims JE, March CJ, Cosman D, Widmer MB, MacDonald HR, McMahan CJ, et al. cDNA expression cloning of the IL-1 receptor, a member of the immunoglobulin superfamily. Science 1988;241:585-9.

28. Cunningham ET Jr, De Souza EB. Interleukin 1 receptors in the brain and endocrine tissues. Immunol Today 1993;14:171-5.

29. Rivier C, Vale W. In the rat, interleukin-1 alpha acts at the level of the brain and the gonads to interfere with gonadotropin and sex steroid secretion. Endocrinology 1989;124:2105-9.

30. Rettori V, Gimeno MF, Karara A, Gonzalez MC, McCann SM. Interleukin-1 alpha inhibits prostaglandin E2 release to suppress pulsatile release of luteinizing hormone but not follicle-stimulating hormone. Proc Natl Acad Sci USA 1991;88:2763-7.

31. Goetzl EJ, Sreedharan SP. Mediators of communication and adaptation in the neuroendocrine and immune systems. FASEB J 1992;6:2646-52.

32. Fukuoka M, Mori T, Taii S, Yasuda K. Interleukin-1 inhibits luteinization of porcine granulosa cells in culture. Endocrinology 1988;122:367-9.

33. Gottschall PE, Katsura G, Hoffmann ST, Arimura A. Interleukin 1: an inhibitor of luteinizing hormone receptor formation in cultured rat granulosa cells. FASEB J 1988;2:2492-6.

34. Nakamura Y, Kato H, Terranova PF. Interleukin-1 alpha increases thecal progesterone production of preovulatory follicles in cyclic hamsters. Biol Reprod 1990;43:169-73.

35. Halme J, Hammond MG, Syrop CH, Talbert LM. Peritoneal macrophages modulate human granulosaluteal cell progesterone production. J Clin Endocrinol Metab 1985;61:912-6.

36. Ebaugh MJ, Smith EM. Human lymphocyte production of immunoreactive luteinizing hormone. FASEB J 1988;2:A1642.

37. Hurwitz A, Loukides J, Ricciarelli E, Botero L, Katz E, McAllister JM, et al. The human intraovarian interleukin-I (IL-I) system: highly compartmentalized and hormonally dependent regulation of the genes encoding IL-I, its receptor, and its receptor antagonist. J Clin Invest 1992;89:1746-54.

38. Polan ML, Kuo A, Loukides J, Bottom L. Cultured human luteal peripheral monocytes secrete increased levels of interleukin- 1. J Endocrinol Metab 1984;70:480-4.

39. Bateman A, Singh A, Kral T, Solomon S. The immunehypothalamic-pituitary-adrenal axis. Endocr Rev 1989;10:92-112. 
40. Cambronero JC, Borrell J, Guaza C. Glucocorticoids modulate rat hypothalamic corticotrophin-releasing factor release induced by interleukin- 1 . J Neurosci Res 1989;24:470-6.

41. Bateman A, Singh A, Kral T, Solomon S. The immunehypothalamic-pituitary-adrenal axis. Endocr Rev 1989;10:92-112.

42. Enomoto T, Sugawa H, Kosugi S, Inoue D, Mori T, Imura H. Prolonged effects of recombinant human interleukin-1 alpha on mouse thyroid function. Endocrinology 1990;127:2322-7.

43. Westermark K, Nilsson M, Karlsson FA. Effects of interleukin 1 alpha on porcine thyroid follicles in suspension culture. Acta Endocrinol (Copenh) 1990;122:505-12.

44. Wiltbank MC, Ottobre JS. Regulation of intraluteal production of prostaglandins. Reprod Biol Endocrinol 2003;1:91.

45. Townson DH, Liptak AR. Chemokines in the corpus luteum: implications of leukocyte chemotaxis. Reprod Biol Endocrinol 2003;1:94.

46. Taub DD. Neuroendocrine interactions in the immune system. Cell Immunol 2008;252:1-6.
47. O'Connor JC, McCusker RH, Strle K, Johnson RW, Dantzer R, Kelley KW. Regulation of IGF-I function by proinflammatory cytokines: at the interface of immunology and endocrinology. Cell Immunol 2008;252:91-110.

48. Lago R, Gómez R, Lago F, Gómez-Reino J, Gualillo O. Leptin beyond body weight regulation-current concepts concerning its role in immune function and inflammation. Cell Immunol 2008;252:139-45.

49. Speroff L, Fritz M. Endocrinología ginecológica clínica y esterilidad. Capítulo 5, 7ª ed; 2007. p.155.

50. Taub DD. Novel Connections between the neuroendocrine and immune systems: the ghrelin immunoregulatory network. Vitam Horm 2008;77:325-46.

51. Webster Marketon JI, Glaser R. Stress hormones and immune function. Cell Immunol 2008;252:16-26.

52. Dmowski WP. Immunological aspects of endometriosis. Int J Gynaecol Obstet 1995;50 Suppl 1:S3-10.

53. Tuohy VK, Altuntas CZ. Autoimmunity and premature ovarian failure. Curr Opin Obstet Gynecol 2007;19:366-9. 